\title{
The Effect of Eccentric Exercise-Induced Delayed-Onset Muscle Soreness on Positioning Sense and Shooting Percentage in Wheelchair Basketball Players
}

\author{
Mehmet Akif Serinken", Celal Gençoğlu², Berkant Muammer Kayatekin¹ \\ ${ }^{1}$ Department of Physiology, Dokuz Eylül University Faculty of Medicine, Izmir, Turkey \\ ${ }^{2}$ Department of Coaching Education, Dokuz Eylül University School of Sport Sciences and Technology, Izmir, Turkey
}

\begin{abstract}
Background: Eccentric exercise is defined as a type of exercise in which the muscle produces power by extending. In contrast to isometric and concentric exercises, eccentric muscle activity is much more effective mechanically; however, it may expose the muscle to soreness. Delayed-Onset Muscle Soreness (DOMS) emerges a couple of hours after an eccentric activity, especially in individuals who are not used to this kind of exercise, and causes a temporary decrease in muscle performance, joint movement angle and muscle power, and also a temporary increase in the blood creatine kinase (CK) activity.

Aims: This study investigates the effect of DOMS on the upper extremities motor performance by conducting an eccentric exercise load on the elbow flexor muscles.

Study design: Cross sectional study.

Methods: The study included 10 wheelchair basketball players. First, the participants underwent blood CK activity, positioning sense, muscle pain, shooting performance measurements tests at the base, and after 30 minutes and 24 and 48 hours. Then, one week later, the one-repetition-maximums of biceps curls were determined in order to define the intensity of the eccentric exercise. An eccentric exercise protocol which would cause DOMS was applied to all players. All tests were replaced with acute exhaustive eccentric exercise; the same tests were repeated in the same order after the exercise. Blood CK activity was measured by taking an earlobe capillary blood sample. The muscle pain level was measured by using a Visual Analogue Scale (VAS). Positioning sense loss was assessed via goniometer at $30^{\circ}, 60^{\circ}$ and $90^{\circ}$ degrees horizontally.
\end{abstract}

Results: The study found a statistically significant increase in blood CK activity and positioning sense loss, and a decrease in the pressure-pain threshold, as well as the shooting percentages in the exercise group when compared with the control.

Conclusion: These findings suggest that DOMS negatively affects the upper extremities motor performance of wheelchair basketball players at least 48 hours after eccentric exercise.

Key Words: Delayed-onset muscle soreness, wheelchair basketball, positioning sense, shooting percentage

Received: 19.12 .2012

Accepted: 05.06.2013

Available Online Date: 27.09.2013

\section{Introduction}

Eccentric exercise is defined as a type of exercise in which the muscle produces power by extending (1). In contrast to isometric and concentric exercises, eccentric muscle activity is much more effective mechanically; however, it may expose the muscle to soreness $(2,3)$. Eccentric contraction either provides a reinforcement stimulus to the skeleton muscles or causes damage to the muscle and produces pain. DelayedOnset Muscle Soreness (DOMS) defines muscle pain and sensitivity which emerges a couple of hours after eccentric muscle movements, especially when the individual is not used to it. At the same time, DOMS is a muscle damage symptom which occurs after eccentric exercise; however, it is not always observed together with muscle damage (4). In the skeleton muscles, the level of muscle proteins such as creatine kinase (CK) increases together with DOMS after heavy exercise, and joint movement angle, muscle power and performance decreases (5). Most studies on DOMS have focused on the plasma CK activity changes, electromyography findings and muscle power, while the effects of DOMS on other components of upper extremities such as coordination and motor performance have been seldom addressed (6).

The upper extremity pain prevalence in manual wheelchair users has been reported in a number of studies; however, few researchers studied the relation of pain to the functional activities (7). In wheelchair basketball players, post-injury traumas have been mostly studied (8). No study of DOMS in wheelchair basketball players could be found in the literature. In this study, the effect of DOMS on upper extremity motor performance was investigated by evaluating the blood CK activity, positioning sense, muscle pain and shooting performance parameters of eccentric exercise in wheelchair basketball players.

\section{Material and Methods}

Ten male wheelchair basketball players who complied with the acceptance criteria volunteered to participate in the 
Table 1. Age, years of basketball playing and player handicap points (average \pm SD)

\begin{tabular}{|lcccc|}
\hline & Minimum & Maximum & Average & Standard Deviation \\
\hline Age (years) & 19 & 46 & 32.6 & \pm 7.61 \\
Basketball Experience (years) & 1 & 22 & 11.9 & \pm 7.34 \\
Player Handicap Points & 1.5 & 4 & 2.75 & \pm 1.0 \\
\hline
\end{tabular}

study. The study was approved by the Dokuz Eylül University Faculty of Medicine Clinic and Laboratory Research's Ethics Committee; the players also provided written informed consent. Players who had any illnesses or reported any upper extremity pain or sports injury on the first day of the study were not included. The players were asked not to use anti-inflammatory drugs and to avoid exhaustive exercises.

First, all participants underwent blood CK activity, positioning sense, muscle pain, shooting performance measurements tests at the base, and after 30 minutes, and 24 and 48 hours (control measurement). Then, one week later, the one-repetition-maximum of the biceps curl was determined in order to define the intensity of the eccentric exercise. An eccentric exercise protocol which would cause DOMS was applied to all players. All tests were replaced with acute exhaustive eccentric exercise (exercise measurement), and the same tests were repeated in the same order after exercise. Player handicap points were used according to the International Wheelchair Basketball Federation (IWBF) Player Classification Commission report and are presented in Table 1 (9).

\section{Blood CK activity}

Blood CK activity was measured by taking earlobe capillary blood samples, as defined by Horder et al. (10). The earlobe was cleaned with alcohol and a $30 \mu \mathrm{L}$ blood sample was taken into a heparinised capillary tube. The sample was taken rapidly from the capillary tube onto the probe and the CK measurement was conducted using the colorimetric measurement procedure (Reflotron Plus, Roche Diagnostic, Mannheim, Germany).

\section{Positioning sense loss measurement}

The measurements were conducted in the dominant upper extremities where eccentric exercise protocol had been applied. The measurements were done on the wheelchairs used by the players during their match using a digital goniometer (Guymon, Model 1129, Lafayette Instruments Co., Lafayette, IN, USA) and a universal goniometer. The universal goniometer was attached to the reference arm, and the digital goniometer was attached to the exercise arm using bandages. Both goniometers were placed, taking the lateral epicondyl of humerus as a pivot, parallel to the static arm humerus lateral midline and towards the stiloid protrusion of the radius of the moving arm, in order to follow the lateral midpoint of the radius. The measurement was conducted while the players were in a seating position in their wheelchairs, with their eyes closed and holding their forearm at three different degrees: $30^{\circ}, 60^{\circ}$ and $90^{\circ}$ degrees horizontally. The reference arm was randomly placed at one of these angles and the player was asked to perform a 2 second isometric flexion contraction at this angle. After contraction, the player was asked to release his arm and position the other arm at the same angle. After a 30 second rest period, this method was repeated at all three degrees, three times each, for a total of 9 trials. The average deviations for each 3 degrees were used as the positioning sense loss data (11).

\section{Muscle pain level measurement}

The measurements were conducted in the dominant upper extremity on which the eccentric exercise protocol had been applied. In order to determine the muscle pain level of the players, the Visual Analogue Scale (VAS) was used. The players were asked to mark the level of muscle pain on a 100 $\mathrm{mm}$ horizontal line. According to this scale, " 0 " indicated that there was no pain and "100" indicated the sharpest pain. Firstly, the players were asked to mark the muscle pain level on VAS with an $(\mathrm{x})$ when their arm was beside their body in its rest position. Later, they were asked to mark the level of the pain on the scale while their elbow joint was in $90^{\circ}$ flexion, in other words when the muscle was in isometric contraction; finally, they were asked to mark the level of the pain when the most bulging part of the muscle was palpated (12).

\section{Determining the shooting percentage}

The players were requested to throw 10 free throws from the free throw line to a standard height hoop after a warming period. The percentage of successful throws was calculated and used as data.

\section{Determining the muscle strength}

Muscle power was determined using the 1-repetition maximum (1RM) according to Baechle and Earle (13). The players exercised for approximately for one minute with a dumbbell to warm up the elbow flexors. The players were asked to make a single elbow flexion. The free weight was adjusted to a greater weight and the players were asked to make an elbow flexion again. This procedure was repeated until the players could not lift the free weight. The last successful lift was recorded as the 1RM value.

\section{Eccentric exercise protocol}

In order to create Delayed-Onset Muscle Soreness, the players completed 4 sets of eccentric exercise, each comprising 20 repetitions of lifting $80 \%$ of their $1 \mathrm{RM}$. The exercise protocol was applied to the dominant upper extremity by using a dumbbell in the wheelchairs. Players' shoulders were supported and their elbows were positioned to $90^{\circ}$ flexion. The players were asked to drop the dumbbell on the ground as such each repetition would end in 2 to 3 seconds. For the 


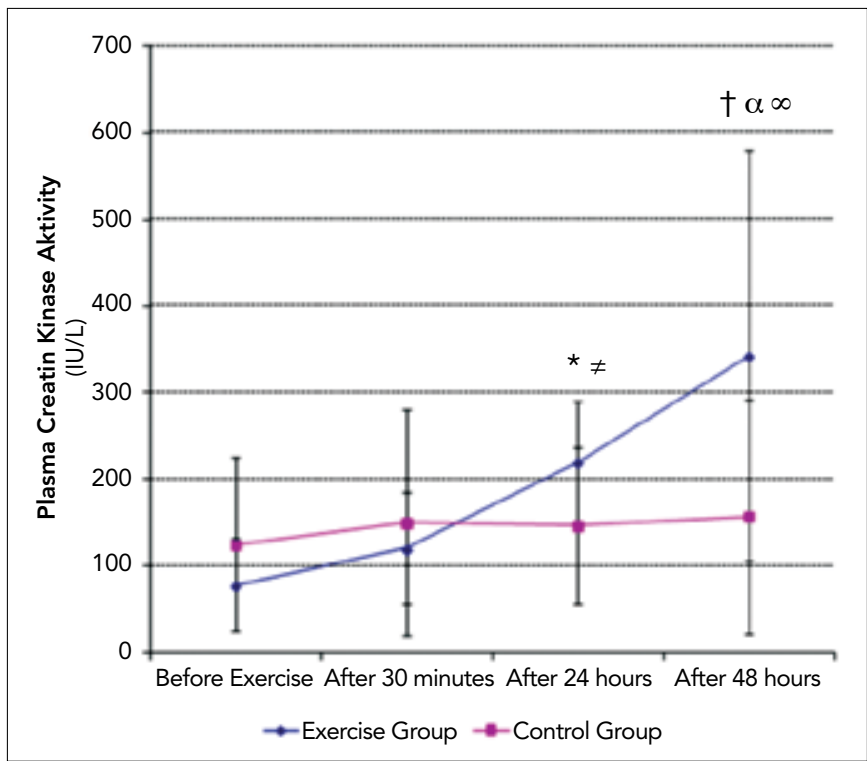

Figure 1. Blood creatine kinase activity $(\mu \mathrm{L})$ (values represent mean and \pm S.D.)

* Higher than before exercise $p<0.05$

\# Higher than after 30-minutes $p<0.05$

$\dagger$ Higher than before exercise $p<0.05$

$\alpha$ Higher than after 30-minutes $p<0.05$

$\infty$ Significantly different between control and exercise measurements after 48-hours $p<0.05$

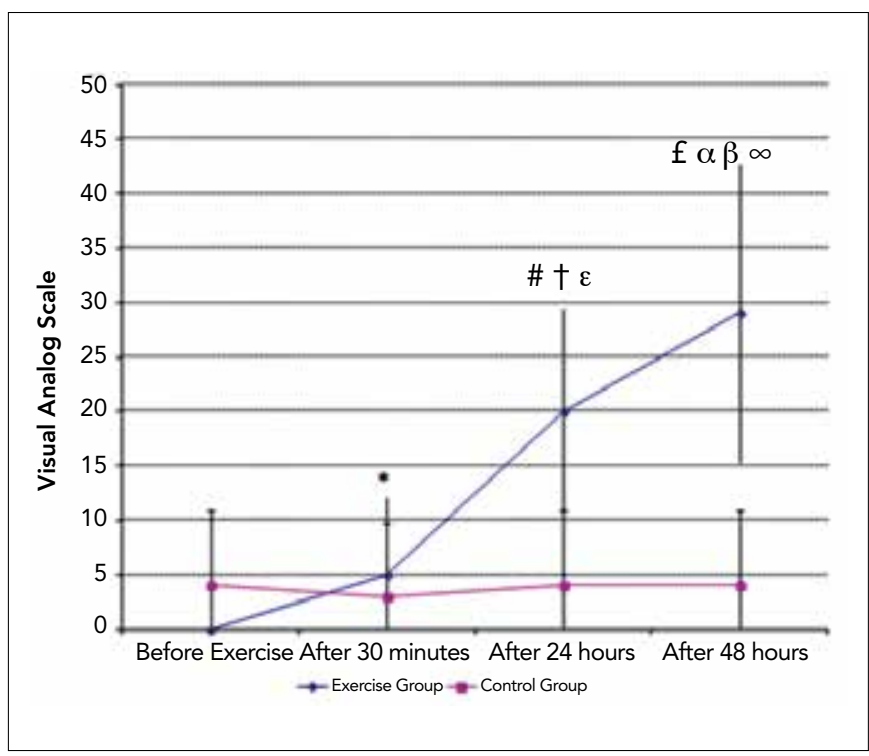

Figure 2. Muscle pain changes at elbow flexion (values represent mean and \pm S.D.)

* Higher than before exercise $p<0.05$

\# Higher than after 30 minutes $p<0.05$

† Higher than before exercise $p<0.05$

$\varepsilon$ Significantly difference between control and exercise measurements after 24-hours $\mathrm{p}<0.01$

$f$ Higher than before exercise $p<0.05$

$\alpha$ Higher than after 30 minutes $p<0.05$

$\beta$ Higher than after 24-hours $p<0.05$

$\infty$ Significantly different between control and exercise measurements after 48-hours $\mathrm{p}<0.01$

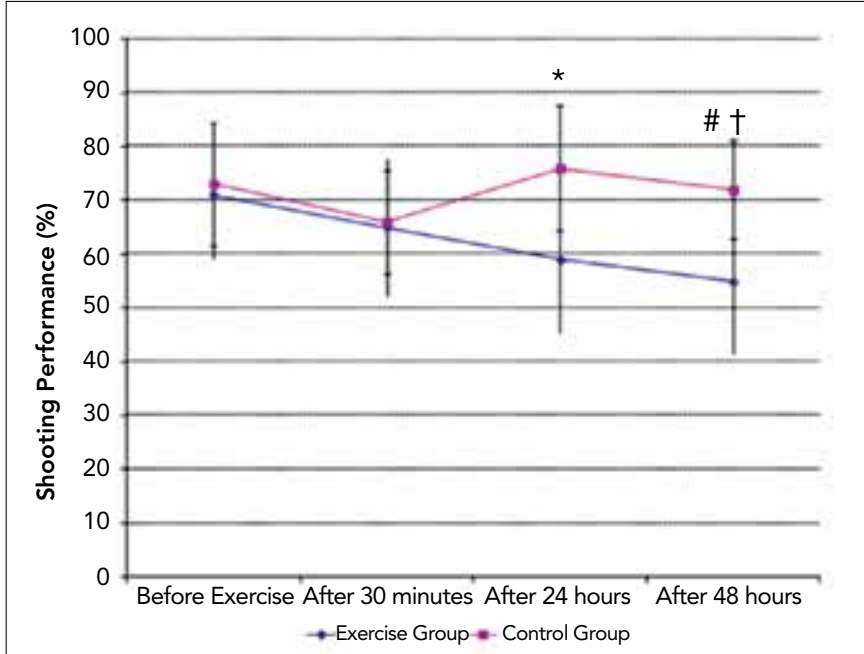

Figure 3. Shooting performance (values represent mean and \pm S.D.)

* Significantly different between control and exercise measurements after 24-hours $p<0.05$

\# Higher than before exercise $p<0.05$

† Significantly different between control and exercise measurements after 48-hours $p<0.05$

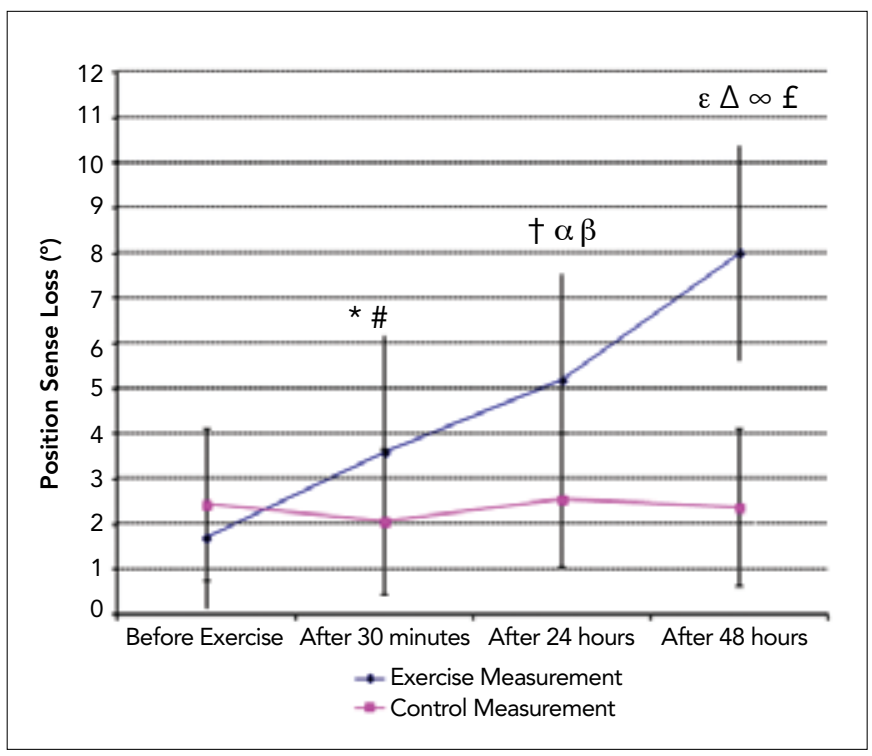

Figure 4. Position sense loss $\left(^{\circ}\right)$ (values represent mean and \pm S.D.)

* Higher than before exercise $p<0.05$

\# Significantly different between control and exercise measurements at after 30 -minutes $p<0.01$

† Higher than 30-minutes $p<0.05$

$\alpha$ Higher than before exercise $p<0.05$

$\beta$ Significantly different between control and exercise measurements after 24-hours $p<0.01$

$\varepsilon$ Higher than before exercise $p<0.05$

$\infty$ Higher than after 30 -minutes $p<0.05$

$\triangle$ Higher than after 24-hours $p<0.05$

$f$ Significantly different between control and exercise measurements after 48-hours $p<0.01$ 
next repetition the dumbbell was brought to the starting position by the researcher (14).

\section{Statistical analysis}

The results were analysed using SPPS (SPSS Inc., Chicago, IL, USA, version 16.0). Friedman Variance Analysis was used to determine the significance within the groups. After the variance analysis, in cases where a significant difference was determined, the Multiple Comparison Test was performed using the Formula below. This calculation shows the difference of repeated measures for non-parametric data and corrects Type 2 errors (15). The statistical significance level was accepted as $p<0.05$.

$$
|R j-R i|\rangle t_{1-\alpha / 2}\left[\frac{2 n\left(A_{2}-B_{2}\right)}{(n-1)(b-1)}\right]^{\frac{1}{2}}
$$

\section{Results}

There were no significantly difference control measurements of blood CK activity. However, after eccentric exercise intervention, blood CK activity significantly increased after 24 hours with respect to the $30^{\text {th }} \mathrm{min}$ and before exercise. Also, there was statistically significant elevation of blood CK levels after 48 hours when compared to the $30^{\text {th }} \mathrm{min}$ and before exercise (Figure 1).

Muscle pain level was determined using VAS and is presented as mean and standard deviation in Figure 2. No change in muscle pain was observed for the control measurements. There was a significant difference for $30^{\text {th }}$ min muscle pain when compared to before exercise. Also, muscle pain was significantly higher in the $24^{\text {th }}$ hour than after $30 \mathrm{~min}$ and before exercise. Furthermore, it was significantly higher after 48 hours than for all prior measurements (Figure 2).

After the eccentric exercise there was a significant difference between control and exercise measurements after the $24^{\text {th }}$ and $48^{\text {th }}$ hours. Shooting performance was decreased after 48 hours when compared to before exercise by $16 \%$ (Figure 3 ).

In exercise measurements, a trend to higher position sense loss was seen for repeated measurements. Furthermore, there was a significantly increased position sense loss for all phases of data when compared with control measurements (Figure 4).

\section{Discussion}

The results of this study indicated that eccentric exercise induced DOMS and position sense loss; also, muscle pain of the elbow flexors increased and shooting performance decreased in wheelchair basketball players. The observed increase in blood CK activity was attributed to DOMS. Our findings are in agreement with earlier researches which showed similar increases in plasma CK activity after eccentric exercise $(16,17)$. It has been mentioned in the literature that soft tissue injuries such as contusions, distention and tearing are the most frequent injuries, while fractures and dislocations are less likely (18). Stohr and Zimmer (19) determined in a study conducted on wheelchair basketball players that $60 \%$ of injuries and overload syndrome occur; $75 \%$ of these are in the upper extremities and $58 \%$ of the injuries are acute. In another study
Burnham et al. (20) reported that $87 \%$ of the wheelchair basketball players were subject to upper extremity injuries and that the players could not continue the matches or the exercises in $18 \%$ of these injuries. As most studies of DOMS and athletic performance being carried out in healthy athletes, no research has been found for wheelchair basketball players. To our knowledge, this is the first report indicating that DOMS has a negative effect on wheelchair basketball shooting performance. It was suggested that any lower extremity force is not used in wheelchair basketball players during shooting, but that the force is created in the body and the upper extremities (21). In another study, Malone et al. (22) stated that deviations in the ball orbit during targeting and inadequate force are the basic factors in unsuccessful free throws in wheelchair basketball players. Therefore, upper extremity power and technique play an important role in shooting success.

There was a significant increase in positioning sense loss in the $30^{\text {th }}$ min after exercise, and that increase remained through 24 and 48 hours after the exercise. In agreement with our study's findings, Saxton et al. (23) recorded significant positioning sense loss immediately after the load on the elbow joint flexion muscles. Similarly, Walsh et al. (24) concluded significant positioning sense loss when the subjects were asked to match positioning of the forearm muscles after an eccentric exercise protocol.

In the present study, when the muscle pain level was tried to be determined by palpating the muscle, a significant increase in muscle pain level in the distal portion of the muscle was found. The muscle pain which occurred immediately after the eccentric exercise is confirmed with by the study of Micklewright et al. (14).

Furthermore, Friden et al. (25) have reported that the increase in the muscle pain level could decrease the muscle power. The findings of our study indicated a significant increase of muscle pain during upper extremity flexion at 30 minutes, 24 hours and 48 hours after the eccentric exercise. Therefore, this muscle pain could affect muscle power, and the shooting percentage as a result. In this study, shooting percentage showed a significant decrease after eccentric exercise. Based upon this finding, it can be argued that DOMS negatively affects the shooting percentage, which is a performance parameter in wheelchair basketball players.

In conclusion; this study presents DOMS, which occurs after eccentric exercise, causes positioning sense loss and a decrease in shooting percentages in wheelchair basketball players. The periodisation of the eccentric exercises must consider the long recovery period required before the match. Also, the time required for the DOMS effects to disappear could not be determined since the study was completed in a 48 hour timeframe. Further studies are needed to reveal the relationship between eccentric exercise and DOMS.

Ethics Committee Approval: This study was approved by Dokuz Eylül University Faculty of Medicine Clinic and Laboratory Researchs Ethical Committee. Date: 23.07.2009 No: 221/2009.

Informed Consent: Written informed consent was obtained from the participants of the study.

Peer-review: Externally peer-reviewed. 
Author contributions: Concept-M.A.S., B.M.K., C.G.; Design-M.A.S., B.M.K., C.G.; Supervision - M.A.S., B.M.K., C.G.; Resource - M.A.S., B.M.K., C.G.; Materials - M.A.S., B.M.K., C.G.; Data Collection\&/or Processing - M.A.S., B.M.K., C.G.; Analysis\&/or Interpretation - M.A.S., B.M.K., C.G.; Literature Search - M.A.S., B.M.K., C.G.; Writing - M.A.S., B.M.K., C.G.; Critical Reviews - M.A.S., B.M.K., C.G.

Conflict of Interest: We certify that there is no actual or potential conflict of interest in relation to this article.

Financial Disclosure: This research funded by Dokuz Eylül University Department of Scientific Research Projects with no: 2009.KB.SAG.061.

\section{References}

1. Barnes PM. Exercise-Induced muscle soreness following high intensity eccentric weightlifting. Master of Science in Physical Education. Health and Human Services California State University, California, 1996.

2. Clarkson PM, Nosaka K, Braun B. Muscle function after exerciseinduced muscle damage and rapid adaptation. Med Sci Sports Exerc 1992;24:512-20. [CrossRef]

3. Enoka RM. Eccentric contractions require unique activation strategies by the nervous system. J Appl Physiol 1996;81:2339-46.

4. Zainuddin Z, Newton M, Sacco P, Nasoka K. Effects of massage on delayed-onset muscle soreness, swelling and recovery of muscle function. J Athl Trai 2005;40:174-80.

5. Howatson G, Van Someren KA. Ice massage: Effects on exercise-induced muscle damage. J of Sports Med Phys Fitness 2003;43:500-5.

6. Kaurenen K, Siira P, Vanharanga H. Delayed-onset muscle soreness and motor performance of the upper extremity. Eur J Appl Physiol 2001;84:302-9. [CrossRef]

7. Curtis KA, Drysdale GA, Lanza D, Kolber M. Shoulder pain in wheelchair users with tetraplegia and paraplegia. Arch Phys Med Rehabil 1999;80:453-7. [CrossRef]

8. Ferrara M S, Peterson CL. Injuries to athletes with disabilities. Sports Med 2000;30:137-43. [CrossRef]

9. IWBF Player Classification Commission. A Guide to the IWBF Functional Classification System for Wheelchair Basketball Players, December 2004:4-9.

10. Hørder M, Jørgensen PJ, Hafkenscheid JC, Carstensen CA, Bachmann C, Bauer K. Creatine kinase determination: a European evaluation of the creatine kinase determination in serum, plasma and whole blood with the Reflotron system. Eur J Clin Chem Clin Biochem 1991;29:691-6.

11. Brockett C, Warren N, Gregory JE, Morgan DL, Proske U. A comparison of the effects of concentric versus eccentric exercise on force and position sense at the human elbow joint. Brain Res 1997;771:251-8. [CrossRef]

12. Vincent HK, Carlson C, Hyatt JP, Yihua L, Vincent KR. Alterations in bilateral force judgment following strenuous eccentric exercise. Res Q Exerc Sport 2000;71:340-8. [CrossRef]

13. Baechle TR, Earle RW. Essentials of strength training and conditioning. 2nd ed. Human Kinetics, Champaign 2000.

14. Micklewright $D$. The effect of soft tissue release on delayed onset muscle soreness: A pilot study. Phys Ther Sport 2009;10:19-24. [CrossRef]

15. WJ Conover. Practical Nonparametric Statistics 2ed p:300-302, Wiley\&Sons 1980

16. Howatson G, Van Someren KA. The prevention and treatment of exercise-induced muscle damage. Sports Med 2008;38:483-503 [CrossRef]

17. Warren GL, Lowe DA, Armstrong RB. Measurement tools used in the study of eccentric contraction-induced injury. Sports Med 1999;27:43-59. [CrossRef]

18. Chapireau F, Colvez A. Social disadvantage in the international classification of impairments, disabilities and handicap. Pergamon 1998;47:59-66.

19. Stohr H, Zimmer M. Wheelchair basketball from the orthopedic viewpoint. Sportverletz Sportschaden 1997;11:109-15.

20. Burnham RS, Higgins J, Steadward RD. Wheelchair basketball injury. Palaestra 1994:10:43-9.

21. Owen E. Playing and coaching wheelchair basketball. Urbana, IL: University of Illinois Press, 1982;35-40.

22. Saxton JM, Clarkson PM, James R, Miles M, Westerfer M, Clark $S$, et al. Neuromuscular dysfunction following eccentric exercise. Med Sci Sports Exerc 1995;27:1185-93. [CrossRef]

23. Walsh LD, Hesse CW, Morgan DL, Proske U. Human forearm position sense after fatigue of elbow flexor muscles. J Physiol 2004;558:705-15. [CrossRef

24. Malone L, Nielsen A, Steadward R. Expanding the dichotomous outcome in wheelchair basketball shooting of elite male players. Adapt Phys Activ Q 2000;17:437-49.

25. Friden J, Lieber RL. Eccentric exercise-induced injuries to contractile and cytoskeletal muscle fibre components. Acta Physiol Scand 2001;171:321-6. [CrossRef] 